\title{
Clinicopathological Features of Adenoid Cystic Carcinoma: An Institutional Perspective
}

\author{
Ushna Mansoor ${ }^{1}$, Atif Ali Hashmi ${ }^{1}$, Naveen Faridi ${ }^{*}$ and Shakil Aqil ${ }^{2}$ \\ ${ }^{1}$ Department of Histopathology, Liaquat National Hospital and Medical College, Karachi, Pakistan \\ ${ }^{2}$ Department of Otorhinolaryngology Head and Neck Surgery, Liaquat National Hospital and Medical College, Karachi, Pakistan
}

\begin{abstract}
Introduction: Adenoid cystic carcinoma is a rare salivary gland malignancy that can occur in any part of the body, its clinicopathologic features are scarcely reported in locoregional literature. Therefore, in this study we aimed to evaluate the clinicopathologic features of adenoid cystic carcinoma in our population and to determine the association of age and site of its occurrence with histologic pattern and perineural invasion.
\end{abstract}

Methods: We retrospectively analyzed 30 cases of adenoid cystic carcinoma that underwent surgical resections or biopsies from March 2009 to February 2020 at Liaquat National Hospital and Medical College, Karachi. Clinical and histopathological parameters including age, site, histological pattern and perineural invasion were determined and association between different parameters was analyzed.

Results: Mean age of the patients was 30+16.1 years. Cribriform pattern was most common histologic pattern (63.3\%) while head and neck region was the most common site of occurrence (83.3\%). Perineural invasion which is one of the most important prognostic factors in adenoid cystic carcinoma was present in approximately half of the patients (46.7\%). No significant association of age and site was found with histologic pattern and perineural invasion.

Conclusion: Adenoid cystic carcinoma is a rare malignancy most commonly occurring in head and neck region with a high frequency of perineural invasion. No significant difference of histologic pattern and histologic prognostic parameter like perineural invasion was noted at different ages and different sites in our study.

Keywords: Adenoid cystic carcinoma, perineural invasion, head and neck malignancy.

\section{INTRODUCTION}

Adenoid cystic carcinoma (ACC) is a rare malignant neoplasm with an indolent nature that shows slow growth but has a tendency for local recurrence and distant metastasis with unfavorable outcome and poor prognosis [1]. It accounts for $1 \%$ of all malignant tumors of head and neck, $22 \%$ of salivary gland malignancies and is commonest malignant tumor of submandibular and minor salivary gland. It usually affects middle aged adults with female predominance [2, 3].

ACC was initially described as Cylindroma by Billroch in 1856. Later it was called ACC of salivary gland by Ewing in 1919, after which the name was applied to tumors of breast by Geschickter in 1945 and that led to its search in other organs as well [4]. ACC arises most commonly in the salivary glands of head and neck region, but has a tendency to originate in any site with secretory glands like lacrimal glands, auditory canal, breast, skin, digestive tract and lungs [5-8].

Although clinicopathologic features of this rare malignancy is described in literature, but apart from a few case reports, studies elaborating this disease lack from our population. Therefore, in this study we aimed

*Corresponding Author: Naveen Faridi, Department of Histopathology, Liaquat National Hospital and Medical College, Karachi, Pakistan;

Email: naveenfaridi@hotmail.com

Received: June 13, 2020; Revised: June 24, 2020; Accepted: June 30, 2020

DOI: https://doi.org/10.37184/lntbj.2708-7808.2.2 to evaluate the clinicopathologic features of adenoid cystic carcinoma in our population and to determine the association of age and site of its occurrence with histologic pattern and perineural invasion.

\section{METHODS}

We retrospectively analyzed 30 cases of adenoid cystic carcinoma that underwent surgical resections or incisional biopsies from March 2009 to February 2020 at Liaquat National hospital and Medical College, Karachi. Cases with prior radiation and chemotherapy were excluded from the study. All specimens were received in Histopathology Department, Liaquat National Hospital and grossly examined according to standard protocols. After overnight processing, hemotoxylin and eosin slides were prepared and examined by senior histopathologists. Immunohistochemical stains including CD117 and p63 were done where necessary. Clinical and histopathological parameters including age, site, histological pattern and perineural invasion were determined and recorded (Figs. $1 \& 2$ ).

Statistical package for social sciences (SPSS 21) was used for data compilation and analysis. Mean and standard deviation were calculated for quantitative variable age. Frequency and percentage were calculated for qualitative variables. Fisher's exact test was applied to determine the association. $p$-value $\leq 0.05$ was considered as statistically significant. 

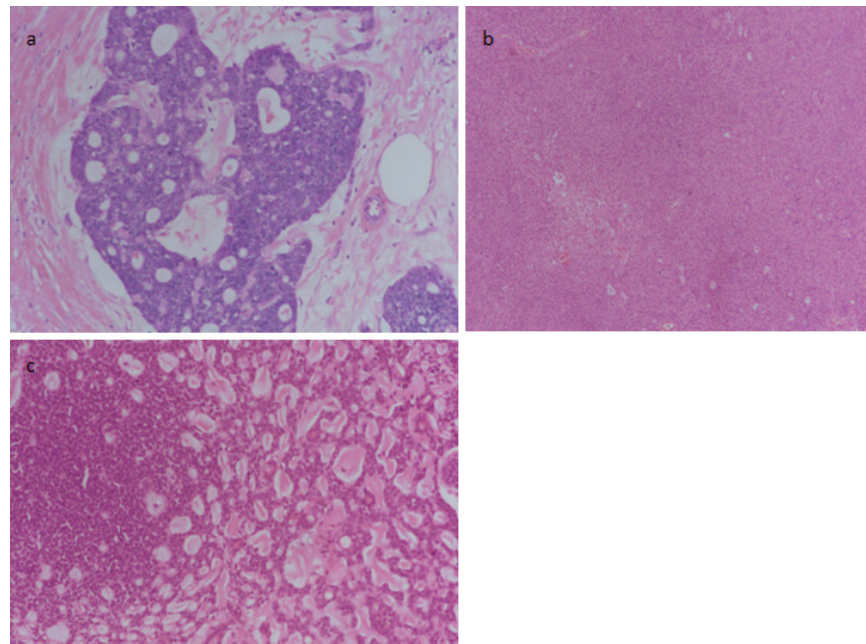

Fig. (1): Different histologic patterns of Adenoid cystic carcinoma a: Cribriform, b: Solid, c: Mixed.

\section{RESULTS}

Mean age of the patients was $30+16.1$ years with range of 9 to 70 years. Most of the patients were between age of $25-50$ years $(n=19,63.3 \%)$. p63 and CD117 was positive in all the cases of ACC. Cribriform pattern was most common histologic pattern $(n=19,63.3 \%)$ while head and neck region was the most common site of occurrence $(n=25,83.3 \%)$. Perineural invasion that is one of the most important prognostic factors in adenoid cystic carcinoma was present in approximately half of the patients $(n=14,46.7 \%)$. Clinicopathologic characteristics of the studied population are presented in Table 1.

Table 1: Clinicopathological characteristics of adenoid cystic carcinoma in studied population.

\begin{tabular}{l|c|c}
\hline Variables & Frequency & Percentage \% \\
\hline Gender & 23 & $76.7 \%$ \\
\hline Female & 7 & $23.3 \%$ \\
\hline Male & 3 & $10.0 \%$ \\
\hline Age & 16 & $53.3 \%$ \\
\hline$<=25$ Years & 11 & $36.7 \%$ \\
\hline $26-50$ Years & 19 & $63.3 \%$ \\
\hline$>50$ Years & 10 & $33.3 \%$ \\
\hline Pattern & 1 & $3.3 \%$ \\
\hline Cribriform & \multicolumn{3}{|l}{} \\
\hline Mixed & 4 & $13.3 \%$ \\
\hline Solid & 1 & $3.3 \%$ \\
\hline Site & 25 & $83.3 \%$ \\
\hline Breast & 16 & $53.3 \%$ \\
\hline Lung & 14 & $46.7 \%$ \\
\hline Head And Neck & \multicolumn{1}{|c}{} \\
\hline Perineural Invasion & Absent &
\end{tabular}

No significant association of age was noted with histologic pattern $(p=1.00)$ and perineural invasion $(p=0.877)$. Similarly, no significant association of site of occurrence with histologic pattern $(p=1.00)$ and perineural invasion was noted $(p=0.602)$ as shown in Table 2.

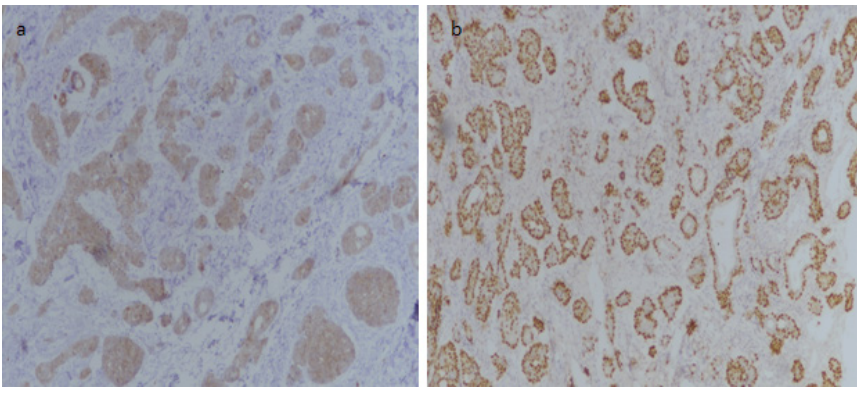

Fig. (2): Immunohistochemical stains in adenoid cystic carcinoma, a: CD117 showing diffuse cytoplasmic and membranous staining, b: p63 showing nuclear staining in myoepithelial component.

Table 2: Association of age and site of occurrence of Adenoid cystic carcinoma with perineural invasion and histologic pattern.

\begin{tabular}{|c|c|c|c|c|c|c|c|}
\hline Variables & \multicolumn{3}{|c|}{ Perineural Invasion } & \multicolumn{4}{|c|}{ Histologic Patterns } \\
\hline Age (Years) & $\begin{array}{c}\text { Absent } \\
\mathrm{n}(\%)\end{array}$ & $\begin{array}{c}\text { Present } \\
\mathrm{n}(\%)\end{array}$ & $p$-value & $\begin{array}{c}\text { Cribriform } \\
\mathrm{n}(\%)\end{array}$ & $\begin{array}{l}\text { Solid } \\
\mathrm{n}(\%)\end{array}$ & $\begin{array}{l}\text { Mixed } \\
\mathrm{n}(\%)\end{array}$ & $p$-value \\
\hline$\leq 25$ years & $1(33.3)$ & $2(66.7)$ & \multirow{3}{*}{0.877} & $2(66.7)$ & $0(0 \%)$ & $1(33.3 \%)$ & \multirow{3}{*}{1.00} \\
\hline $26-50$ years & $9(56.2)$ & $7(43.8)$ & & $10(62.5)$ & $1(6.2)$ & $5(31.2)$ & \\
\hline$>50$ years & $6(54.5)$ & $5(45.5)$ & & $7(63.6)$ & $0(0)$ & $4(36.4)$ & \\
\hline \multicolumn{8}{|l|}{ Tumor Site } \\
\hline $\begin{array}{l}\text { Head and } \\
\text { neck }\end{array}$ & $12(48)$ & $13(52)$ & \multirow{3}{*}{0.602} & $15(60)$ & $1(4)$ & $9(36)$ & \multirow{3}{*}{1.00} \\
\hline Lung & $1(100)$ & $0(0)$ & & $1(100)$ & $0(0)$ & $0(0)$ & \\
\hline Breast & $3(75)$ & $1(25)$ & & $3(75)$ & $0(0)$ & $1(25)$ & \\
\hline
\end{tabular}

\section{DISCUSSION}

In the present study we found that ACC commonly occurred in head and neck region with only a few of cases to be present at other sites like lung and breast. Moreover, approximately half of ACC exhibited perineural invasion that is one of the prognostic parameters of ACC [2,3]. We did not find any significant association of age and site of ACC with histologic prognostic parameters like solid pattern and perineural invasion. To our knowledge, this is the first study describing clinicopathologic features of ACC in our population.

ACC is diagnosed late in course of the disease because it tends to show symptoms when it has already locally invaded the organ and has involved the nerves around the invaded region or the tumor is causing compression effects. Although most patients present with locally invasive disease, this malignant neoplasm has the ability of distant metastasis, most commonly to lungs and bone [9]. In our study, follow up of patients was not available to determine frequency of developing recurrence and metastasis.

ACC shows a myoepithelial differentiation composed of dual population of basaloid and luminal cells. Different architectural patterns are reported e.g. cribiform, tubular, trabecular and solid [10]. The basaloid cells show positivity for smooth muscle actin, $\mathrm{S} 100$, vimentin, calpinin and p63 while the epithelial cells show positivity for luminal cytokeratins such as CK7, CEA, EMA, and CD117. In our study most of the cases exhibited cribriform pattern, only a few of cases revealed solid and 
mixed pattern. Although ACC is a histologic diagnosis, we performed p63 and CD117 stains in most cases to confirm the diagnosis.

ACC is a rare malignancy as reflected by a small sample size in most of the previously published international studies [11-13], on the other hand local data is limited to a few case reports and studies involving salivary gland malignancies as a whole. Mean age of the patients in different studies ranges from 51-58 years [10-13]. In contrast our study has mean age of 30 years. This difference may be due to small sample size in our study or different tumor biology in this part of the world. Few studies suggested female gender predominance in ACC [14-16] in contrast to our study. Most of the studies reported cribriform pattern as the commonest histologic pattern and high frequency of perineural invasion especially in head and neck ACC [17, 18]. None of the previously published studies found any significant association of age and site with prognostic parameters like perineural invasion, although solid histologic pattern was considered as marker of poor prognosis and distant metastasis in ACC [19].

This study can be viewed with a few limitations like small sample size and lack of clinical follow up to determine disease free survival and distant metastasis; however, finding of our study can be viewed with a substantial clinical importance as this is a rare disease and current literature on the topic is limited in our population.

\section{CONCLUSION}

Adenoid cystic carcinoma is a rare malignancy most commonly occurring in head and neck region with a high frequency of perineural invasion. No significant association of histologic pattern and perineural invasion was noted with age and site of occurnce of ACC in our study.

\section{CONFLICT OF INTEREST}

The authors declare no conflict of interest.

\section{ACKNOWLEDGEMENTS}

We gratefully acknowledge all staff members of Pathology Department, Liaquat National Hospital, Karachi, Pakistan for their help and cooperation.

\section{REFERENCES}

1. Jaso J, Malhotra R. Adenoid cystic carcinoma. Arch Pathol Lab Med 2011; 135(4): 511-5.
2. Seethala RR, Hunt JL, Baloch ZW, Livolsi VA, Leon Barnes E. Adenoid cystic carcinoma with high-grade transformation: a report of 11 cases and a review of the literature. Am J Surg Pathol 2007; 31(11): 1683-94.

3. Spiro RH. Distant metastasis in adenoid cystic carcinoma of salivary origin. Am J Surg 1997; 174(5): 495-8.

4. Marchiò $C$, Weigelt $B$, Reis-Filho JS. Adenoid cystic carcinomas of the breast and salivary glands (or 'The strange case of Dr Jekyll and Mr Hyde' of exocrine gland carcinomas). J Clin Pathol 2010; 63(3): 220-8.

5. Chaudhry AP, Leifer C, Cutler LS, Satchidanand S, Labay GR, Yamane GM. Histogenesis of adenoid cystic carcinoma of the salivary glands. Light and electronmicroscopic study. Cancer 1986; 58(1): 72-82.

6. Lawrence JB, Mazur MT. Adenoid cystic carcinoma: a comparative pathologic study of tumors in salivary gland, breast, lung, and cervix. Hum Pathol 1982; 13(10): 916-24.

7. Nelms DC, Luna MA. Primary adenocystic carcinoma (cylindromatous carcinoma) of the esophagus. Cancer 1972; 29(2): 440-3.

8. Sarnaik AA, Meade T, King J, et al. Adenoid cystic carcinoma of the breast: a review of a single institution's experience. Breast J 2010; 16(2): 208-10.

9. Locati LD, Guzzo M, Bossi P, et al. Lung metastasectomy in adenoid cystic carcinoma (ACC) of salivary gland. Oral Oncol 2005; 41(9): 890-4

10. Cheuk W, Chan JK. Advances in salivary gland pathology. Histopathology 2007; 51(1): 1-20.

11. DeAngelis AF, Tsui A, Wiesenfeld D, Chandu A. Outcomes of patients with adenoid cystic carcinoma of the minor salivary glands. Int J Oral Maxillofac Surg 2011; 40(7): 710-14.

12. Min R, Siyi L, Wenjun $Y$, et al. Salivary gland adenoid cystic carcinoma with cervical lymph node metastasis: a preliminary study of 62 cases. Int J Oral Maxillofac Surg 2012; 41(8): 952-7.

13. Lukšić I, Suton P, Macan D, Dinjar K. Intraoral adenoid cystic carcinoma: is the presence of perineural invasion associated with the size of the primary tumour, local extension, surgical margins, distant metastases, and outcome? Br J Oral Maxillofac Surg 2014; 52(3): 214-8.

14. Tincani AJ, Del Negro A, Araújo PP, et al. Management of salivary gland adenoid cystic carcinoma: institutional experience of a case series. Sao Paulo Med J 2006; 124(1): 26-30.

15. Khan AJ, DiGiovanna MP, Ross DA, et al. Adenoid cystic carcinoma: a retrospective clinical review. Int J Cancer 2001; 96(3): 149-58.

16. Sung MW, Kim KH, Kim JW, et al. Clinicopathologic predictors and impact of distant metastasis from adenoid cystic carcinoma of the head and neck. Arch Otolaryngol Head Neck Surg 2003; 129(11): 1193-7.

17. Bianchi B, Copelli C, Cocchi R, Ferrari S, Pederneschi N, Sesenna E. Adenoid cystic carcinoma of intraoral minor salivary glands. Oral Oncol 2008; 44(11): 1026-31.

18. da Cruz Perez DE, de Abreu Alves F, Nobuko NI, de Almeida OP, Kowalski LP. Prognostic factors in head and neck adenoid cystic carcinoma. Oral Oncol 2006; 42(2): 139-46.

19. Coca-Pelaz A, Rodrigo JP, Bradley PJ, et al. Adenoid cystic carcinoma of the head and neck-an update. Oral Oncol 2015; 51(7): 652-61. 